\title{
Stability Analysis of Hybrid Systems via Small-Gain Theorems
}

\author{
Daniel Liberzon ${ }^{1 \star}$ and Dragan Nešić ${ }^{2 \star \star}$ \\ 1 Coordinated Science Laboratory, \\ University of Illinois at Urbana-Champaign, \\ Urbana, IL 61801, U.S.A., \\ liberzon@uiuc.edu \\ 2 Department of Electrical and Electronic Engineering, \\ University of Melbourne, \\ Parkville, 3052, Victoria, Australia, \\ d.nesic@ee.unimelb.edu.au
}

\begin{abstract}
We present a general approach to analyzing stability of hybrid systems, based on input-to-state stability (ISS) and small-gain theorems. We demonstrate that the ISS small-gain analysis framework is very naturally applicable in the context of hybrid systems. Novel Lyapunovbased and LaSalle-based small-gain theorems for hybrid systems are presented. An illustrative application of the proposed approach in the context of a quantized feedback control problem is treated in detail. The reader does not need to be familiar with ISS or small-gain theorems to be able to follow the paper.
\end{abstract}

\section{Introduction}

The small-gain theorem is a classical tool for analyzing input-output stability of feedback systems; see, e.g., [1]. More recently, small-gain tools have been used extensively to study feedback interconnections of nonlinear state-space systems in the presence of disturbances; see, e.g., [2].

Hybrid systems can be naturally viewed as feedback interconnections of simpler subsystems. For example, every hybrid system can be regarded as a feedback interconnection of its continuous and discrete dynamics. This makes small-gain theorems a very natural tool to use for studying internal and external stability of hybrid systems. However, we are not aware of any systematic application of this idea in the hybrid systems literature.

The purpose of this paper is to bring the small-gain analysis method to the attention of the hybrid systems community. We review, in a leisurely tutorial fashion, the concept of input-to-state stability (ISS) introduced by Sontag [3]

* Supported by NSF ECS-0134115 CAR and DARPA/AFOSR MURI F49620-02-10325 Awards.

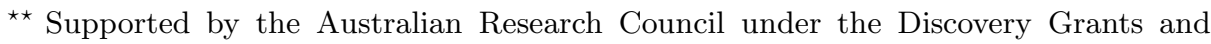
Australian Professorial Fellow schemes. 
and a nonlinear small-gain theorem from [2] based on this concept. The ISS small-gain theorem states that a feedback interconnection of two ISS systems is ISS if an appropriate composition of their respective ISS gain functions is smaller than the identity function. Since a proof of this theorem can be based entirely on time-domain analysis of system signals, the result is valid for general dynamical systems, thus providing an "off-the-shelf" method for verifying stability of hybrid systems. We then discuss Lyapunov-based tools for checking the hypotheses of this theorem.

As an alternative to time-domain proofs, Lyapunov function constructions for interconnected systems under small-gain conditions were suggested for continuoustime systems in [4] and for discrete-time systems in [5]. It is generally well known that having a Lyapunov function provides additional insight into the behavior of a stable system and is important for tasks such as perturbation analysis and estimating the region of attraction. In this paper, we present a novel construction of a Lyapunov function for a class of hybrid systems satisfying the conditions of the ISS small-gain theorem. We also describe another approach, based on constructing a weak (non-strictly decreasing) Lyapunov function and applying the LaSalle invariance principle for hybrid systems from [6]. We note that while the basic idea of the small-gain stability analysis for hybrid systems was announced and initially examined by the authors in [7], the Lyapunov function constructions are reported here for the first time and represent the main technical contribution of this work.

We illustrate the power of the proposed method by analyzing a quantized feedback control strategy previously studied in $[8,9]$ using different tools. Other applications in the area of hybrid control with communication constraints are discussed in [7]. The small-gain analysis is found to provide insightful interpretations of existing results, immediately leads to generalizations, and allows a unified treatment of problems that so far have been studied separately. Due to the pervasive nature of hybrid systems in applications, we expect that the main idea proposed in this paper will be useful in many other areas as well.

\section{Preliminaries}

In what follows, $i d$ denotes the identity function and $\circ$ denotes function composition. We write $a \vee b$ for $\max \{a, b\}$ and $a \wedge b$ for $\min \{a, b\}$. The class of continuously differentiable functions is denoted by $C^{1}$ (the domain will be spec-

ified separately). Given some vectors $x_{1} \in \mathbb{R}^{n_{1}}$ and $x_{2} \in \mathbb{R}^{n_{2}}$, we often use the simplified notation $\left(x_{1}, x_{2}\right)$ for the "stack" vector $\left(x_{1}^{T}, x_{2}^{T}\right)^{T} \in \mathbb{R}^{n_{1}+n_{2}}$.

\subsection{Hybrid system model}

We begin by describing the model of a hybrid system to which our subsequent results will apply. This model easily fits into standard modeling frameworks for hybrid systems (see, e.g., $[10,6,11]$ ), and the reader can consult these references 
for background and further technical details. The description to be provided here is somewhat informal, but it is sufficient for presenting the results.

We label the hybrid system to be defined below as $\mathcal{H}$. The state variables of $\mathcal{H}$ are divided into continuous variables $x \in \mathbb{R}^{n}$, discrete variables $\mu \in \mathbb{R}^{k}$, and additional variables $\tau \in \mathbb{R}^{l}$. We note that $\mu$ actually takes values in a discrete subset of $\mathbb{R}^{k}$ along every trajectory of the hybrid system, but this set need not be fixed a priori and may vary with initial conditions. The variables $\tau$ represent auxiliary states thought of primarily as continuous clocks. The time is continuous: $t \in\left[t_{0}, \infty\right)$. We also consider external variables $w \in \mathbb{R}^{s}$, viewed as disturbances.

The state dynamics describing the evolution of these variables with respect to time are composed of continuous evolution and discrete events. During continuous evolution (i.e., while no discrete events occur), $\mu$ is held constant, $x$ satisfies the ordinary differential equation

$$
\dot{x}=f(x, \mu, w)
$$

with $f: \mathbb{R}^{n} \times \mathbb{R}^{k} \times \mathbb{R}^{s} \rightarrow \mathbb{R}^{n}$ locally Lipschitz, and $\tau$ satisfies

$$
\dot{\tau}_{i}=1, \quad i=1, \ldots, l .
$$

We now describe the discrete events. Given an arbitrary time $t$, we will denote by $x^{-}(t)$, or simply by $x^{-}$when the time arguments are omitted, the quantity $x\left(t^{-}\right)=\lim _{s / t} x(s)$, and similarly for the other state variables. Consider a guard map $G: \mathbb{R}^{n+k+l} \rightarrow \mathbb{R}^{p}$ (where $p$ is a positive integer) and a reset map $R: \mathbb{R}^{n+k+l} \rightarrow \mathbb{R}^{n+k+l}$. The discrete events are defined as follows: whenever

$$
G\left(x^{-}, \mu^{-}, \tau^{-}\right) \geq 0
$$

(component-wise), we let

$$
\left(\begin{array}{l}
x \\
\mu \\
\tau
\end{array}\right)=R\left(x^{-}, \mu^{-}, \tau^{-}\right)=\left(\begin{array}{l}
R_{x}\left(x^{-}, \mu^{-}, \tau^{-}\right) \\
R_{\mu}\left(x^{-}, \mu^{-}, \tau^{-}\right) \\
R_{\tau}\left(x^{-}, \mu^{-}, \tau^{-}\right)
\end{array}\right) .
$$

By construction, all signals are right-continuous.

Some remarks on the above relations are in order. In many situations, $R_{x}(x, \mu, \tau) \equiv$ $x$, i.e., the continuous state does not jump at the event times. We want inequality rather than equality in (1) because for a discrete event to occur, we might need several conditions which do not become valid simultaneously (e.g., some relation between $x$ and $\mu$ holds and a clock has reached a certain value). Of course, equality conditions are easily described by pairs of inequalities. The continuous time $t$ does not explicitly appear in the dynamics. If desired, it could be incorporated either in $x$ (with equation $\dot{t}=1$ ) or in $\tau$, and in either case it is not reset at event times. For simplicity, we assume that the disturbances $w$ affect only the continuous evolution and the auxiliary variables $\tau$ affect only the discrete events, because this is the case in the examples studied below and in [7]. The discrete events in general do not need to occur in any periodic fashion, and 
different kinds of discrete events can happen completely asynchronously. We can have synchronous periodic behavior as a special case, if the discrete events are triggered entirely by a clock reaching a fixed value, after which the clock is reset to 0 .

Well-posedness (existence and uniqueness of solutions) of the hybrid system $\mathcal{H}$ is an issue; see, e.g., [10]. At the general level of the present discussion, we are going to assume it. For example, we can assume that the use of auxiliary variables (clocks) in the reset condition (1) ensures that a bounded number of discrete events occurs in any bounded time interval. Then, to obtain a solution (in the sense of Carathéodory), we simply flow the continuous dynamics until either the end of their domain is reached (finite escape) or a discrete event occurs; in the latter case, repeat from the new state, and so on. This construction will apply in the example treated in Section 6. See also [12] for an interesting alternative definition of solutions of hybrid systems.

\subsection{Feedback interconnection structure}
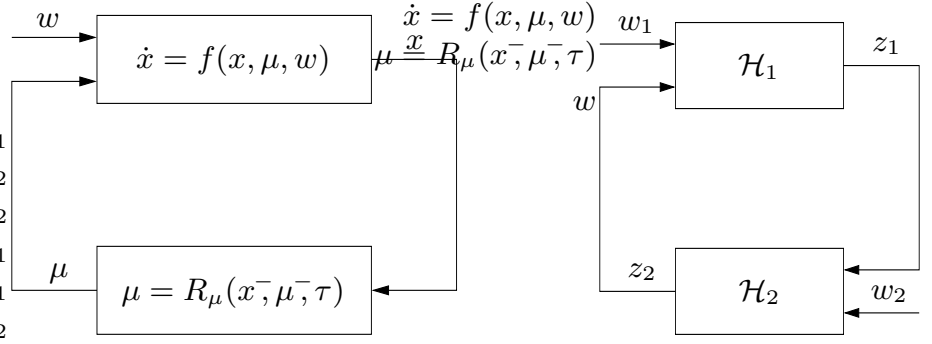

Fig. 1. Hybrid system viewed as feedback interconnection: (a) simple decomposition, (b) general decomposition

The starting point for our results is the observation that we can view the hybrid system $\mathcal{H}$ as a feedback interconnection of its continuous and discrete parts, as shown in Figure 1(a). The auxiliary variables $\tau$ are available to the discrete subsystem (because they are needed to determine the event times and execute resets) and possibly also available to the continuous subsystem. We do not display their dynamics explicitly in the picture because we will not be concerned about their behavior.

It is clear that the above decomposition is just one possible way to split the hybrid system $\mathcal{H}$ into a feedback interconnection of two subsystems. There may be many ways to do it; the best choice will depend on the structure of the problem and will be one for which the small-gain approach will work (see below). Each subsystem in the decomposition can be continuous, discrete, or hybrid, and may be affected by the disturbances. This more general situation is illustrated in Figure 1(b). Here, the state variables and the external signals of $\mathcal{H}$ 
are split as $x=\left(x_{1}, x_{2}\right), \mu=\left(\mu_{1}, \mu_{2}\right), w=\left(w_{1}, w_{2}\right)$, the first subsystem $\mathcal{H}_{1}$ has states $z_{1}=\left(x_{1}, \mu_{1}\right)$ and inputs $v_{1}=\left(z_{2}, w_{1}\right)$, and the second subsystem $\mathcal{H}_{2}$ has states $z_{2}=\left(x_{2}, \mu_{2}\right)$ and inputs $v_{2}=\left(z_{1}, w_{2}\right)$. As we did in the previous example, we can actually omit some variables that are not of immediate interest from the states of the feedback interconnection (these variables would still be used in establishing the desired properties of the two components). Generalization to the case of partial measurements (outputs) is also straightforward.

In the approach discussed here, coming up with an appropriate decomposition of the above kind is the first step in the analysis of a given hybrid system $\mathcal{H}$. As we pointed out, such a decomposition always exists. It can also happen that the hybrid system model is given from the beginning as an interconnection of several hybrid systems. Thus the structure we consider is very general and not at all restrictive.

\subsection{Stability definitions}

A function $\alpha:[0, \infty) \rightarrow[0, \infty)$ is said to be of class $\mathcal{K}($ which we write as $\alpha \in \mathcal{K}$ ) if it is continuous, strictly increasing, and $\alpha(0)=0$. If $\alpha$ is also unbounded, then it is said to be of class $\mathcal{K}_{\infty}\left(\alpha \in \mathcal{K}_{\infty}\right)$. The simplest example of a class $\mathcal{K}_{\infty}$ function is

$$
\alpha(r)=c r, \quad c>0 .
$$

A function $\beta:[0, \infty) \times[0, \infty) \rightarrow[0, \infty)$ is said to be of class $\mathcal{K} \mathcal{L}(\beta \in \mathcal{K} \mathcal{L})$ if $\beta(\cdot, t)$ is of class $\mathcal{K}$ for each fixed $t \geq 0$ and $\beta(r, t)$ is decreasing to zero as $t \rightarrow \infty$ for each fixed $r \geq 0$. The simplest example of a class $\mathcal{K} \mathcal{L}$ function is

$$
\beta(r, t)=c r e^{-\lambda t}, \quad c, \lambda>0 .
$$

We now define the stability notions of interest in this paper. Consider a hybrid system with state $z=(x, \mu)$ and input $v$ (as a special case, it can have only continuous dynamics or only discrete events). Following [3], we say that this system is input-to-state stable (ISS) with respect to $v$ if there exist functions $\beta \in \mathcal{K} \mathcal{L}$ and $\gamma \in \mathcal{K}_{\infty}$ such that for every initial state $z\left(t_{0}\right)$ and every input $v(\cdot)$ the corresponding solution satisfies the inequality

$$
|z(t)| \leq \beta\left(\left|z\left(t_{0}\right)\right|, t-t_{0}\right)+\gamma\left(\|v\|_{\left[t_{0}, t\right]}\right)
$$

for all $t \geq t_{0}$, where $\|v\|_{\left[t_{0}, t\right]}:=\sup \left\{|v(s)|: s \in\left[t_{0}, t\right]\right\}$ (except possibly on a set of measure 0). We will refer to $\gamma$ as an ISS gain function, or just a gain if clear from the context. For time-invariant systems, we can take the initial time to be 0 without loss of generality.

In the case of no inputs $(v \equiv 0)$, the above inequality reduces to

$$
|z(t)| \leq \beta\left(\left|z\left(t_{0}\right)\right|, t\right) \quad \forall t \geq t_{0}
$$

which corresponds to the standard notion ${ }^{3}$ of global asymptotic stability (GAS). In the presence of inputs, ISS captures the property that bounded inputs and

\footnotetext{
${ }^{3}$ This can also be equivalently restated in the more classical $\varepsilon-\delta$ style (cf. [13]).
} 
inputs converging to 0 produce states that are also bounded and converging to 0 , respectively.

If the inputs are split as $v=\left(v_{1}, v_{2}\right)$, then (4) is equivalent to

$$
|z(t)| \leq \beta\left(\left|z\left(t_{0}\right)\right|, t-t_{0}\right)+\gamma_{1}\left(\left\|v_{1}\right\|_{\left[t_{0}, t\right]}\right)+\gamma_{2}\left(\left\|v_{2}\right\|_{\left[t_{0}, t\right]}\right)
$$

for some functions $\gamma_{1}, \gamma_{2} \in \mathcal{K}_{\infty}$. In this case, we will call $\gamma_{1}$ the ISS gain from $v_{1}$ to $z$, and so on.

We note that asymptotic stability of a linear system (continuous or sampleddata) can always be characterized by a class $\mathcal{K} \mathcal{L}$ function of the form (3). Moreover, an asymptotically stable linear system is automatically ISS with respect to external inputs, with a linear ISS gain function $\gamma$ as in (2).

\section{ISS small-gain theorem}

Consider the hybrid system $\mathcal{H}$ defined in Section 2.1, and suppose that it has been represented as a feedback interconnection of two subsystems $\mathcal{H}_{1}$ and $\mathcal{H}_{2}$ in the way described earlier and shown in Figure 1(b). The small-gain theorem stated next reduces the problem of verifying ISS of $\mathcal{H}$ to that of verifying ISS of $\mathcal{H}_{1}$ and $\mathcal{H}_{2}$ and checking a condition that relates their respective ISS gains. The result we give is a special case of the small-gain theorem from [2]. That paper treats continuous systems, but since the statement and the proof given there involve only properties of system signals, the fact that the dynamics are hybrid in our case does not change the validity of the argument. We note that the result presented in [2] is much more general in that it treats partial measurements (input-to-output-stability, in conjunction with detectability) and deals with practical stability notions. Many other versions are also possible, e.g., we can replace the sup norm used in (4) by an $L_{p}$ norm [14].

Theorem 1 Suppose that:

1. $\mathcal{H}_{1}$ is ISS with respect to $v_{1}=\left(z_{2}, w_{1}\right)$, with gain $\gamma_{1}$ from $z_{2}$ to $z_{1}$, i.e.,

$$
\left|z_{1}(t)\right| \leq \beta_{1}\left(\left|z_{1}\left(t_{0}\right)\right|, t-t_{0}\right)+\gamma_{1}\left(\left\|z_{2}\right\|_{\left[t_{0}, t\right]}\right)+\bar{\gamma}_{1}\left(\left\|w_{1}\right\|_{\left[t_{0}, t\right]}\right)
$$

for some $\beta_{1} \in \mathcal{K} \mathcal{L}, \gamma_{1}, \bar{\gamma}_{1} \in \mathcal{K}_{\infty}$.

2. $\mathcal{H}_{2}$ is ISS with respect to $v_{2}=\left(z_{1}, w_{2}\right)$, with gain $\gamma_{2}$ from $z_{1}$ to $z_{2}$, i.e.,

$$
\left|z_{2}(t)\right| \leq \beta_{2}\left(\left|z_{2}\left(t_{0}\right)\right|, t-t_{0}\right)+\gamma_{2}\left(\left\|z_{1}\right\|_{\left[t_{0}, t\right]}\right)+\bar{\gamma}_{2}\left(\left\|w_{2}\right\|_{\left[t_{0}, t\right]}\right)
$$

for some $\beta_{2} \in \mathcal{K} \mathcal{L}, \gamma_{2}, \bar{\gamma}_{2} \in \mathcal{K}_{\infty}$.

3. There exists a function $\rho \in \mathcal{K}_{\infty}$ such that ${ }^{4}$

$$
(i d+\rho) \circ \gamma_{1} \circ(i d+\rho) \circ \gamma_{2}(r) \leq r \quad \forall r \geq 0 .
$$

Then $\mathcal{H}$ is ISS with respect to the input $w=\left(w_{1}, w_{2}\right)$.

\footnotetext{
${ }^{4}$ If one replaces $\beta+\gamma$ with $\max \{\beta, \gamma\}$ in the definition (4) of ISS, then the small-gain condition (5) can be simplified to $\gamma_{1} \circ \gamma_{2}(r)<r$ for all $r>0$.
} 
Three special cases are worth mentioning explicitly, because they will arise in the discussions that follow. First, in the case of no external signals $\left(w_{1}=w_{2} \equiv 0\right)$, we conclude that $\mathcal{H}$ is GAS. Second, when the two ISS gain functions are linear: $\gamma_{i}(r)=c_{i} r, i=1,2$, the small-gain condition (5) reduces to the simple one $c_{1} c_{2}<1$. Third, the theorem covers the case of a cascade connection, where one of the gains is 0 and hence the small-gain condition (5) is automatically satisfied.

As already mentioned, we will sometimes concentrate only on some states of the overall system, excluding the other states from the feedback interconnection. Typically, these underlying "hidden" states have very simple dynamics and remain bounded for all time. Theorem 1 is still valid if we let $z_{1}$ and $z_{2}$ include only the states of interest for each subsystem. ${ }^{5}$

Small-gain theorems have been widely used for analysis of continuous-time as well as discrete-time systems with feedback interconnection structure. The discussion of Section 2.2 suggests that it is also very natural to use this idea to analyze (internal or external) stability of hybrid systems. Of course, we will need to be able to prove that the subsystems in a given feedback decomposition satisfy suitable ISS properties, and calculate the ISS gains in order to check (5). There exist efficient tools for doing this, as exemplified in the next section.

\section{Sufficient conditions for ISS}

Consider the hybrid system $\mathcal{H}$ defined in Section 2.1, and suppose that it has been represented as a special feedback interconnection shown in Figure 1(a). The two lemmas stated below provide Lyapunov-based conditions which guarantee ISS of the continuous and discrete dynamics, respectively, and give expressions for the ISS gains. Thus they can be used for verifying the hypotheses of Theorem 1 in this particular case. The first result is well established [3]; the second one is a slightly improved version of Theorem 4 from the recent paper [16].

Lemma 1 Suppose that there exists a $C^{1}$ function $V_{1}: \mathbb{R}^{n} \rightarrow \mathbb{R}$, class $\mathcal{K}_{\infty}$ functions $\alpha_{1, x}, \alpha_{2, x}, \rho_{x}, \sigma$, and a continuous positive definite function $\alpha_{3, x}:[0, \infty) \rightarrow$ $[0, \infty)$ satisfying

$$
\alpha_{1, x}(|x|) \leq V_{1}(x) \leq \alpha_{2, x}(|x|)
$$

and

$$
V_{1}(x) \geq \rho_{x}(|\mu|) \vee \sigma(|w|) \Rightarrow \nabla V_{1}(x) f(x, \mu, w) \leq-\alpha_{3, x}\left(V_{1}(x)\right) .
$$

Then the $x$-subsystem is ISS with respect to $(\mu, w)$, with gain $\gamma_{x}:=\alpha_{1, x}^{-1} \circ \rho_{x}$ from $\mu$ to $x$.

The condition (6) simply says that $V_{1}$ is positive definite and radially unbounded. We can take $\alpha_{3, x} \in \mathcal{K}_{\infty}$ with no loss of generality [3]. The condition (7) can be equivalently rewritten as

$$
\nabla V_{1}(x) f(x, \mu, w) \leq-\alpha_{4, x}\left(V_{1}(x)\right)+\chi_{x}(|\mu|)
$$

\footnotetext{
5 These modified hypotheses amount to replacing ISS with a suitable input-to-output stability notion (cf. $[2,15])$ and requiring that the ISS gain from the hidden states in each subsystem to the states of interest in the other subsystem be 0 .
} 
for some $\alpha_{4, x}, \chi_{x} \in \mathcal{K}_{\infty}$. However, using this condition instead of (7) in the lemma would in general lead to a more conservative ISS gain. We also note that Lemma 1 can be easily generalized by allowing $V_{1}$ to depend on $t$ as well as on $x$, leaving the bounds in (6) unchanged, and adding the time derivative of $V_{1}$ in (7); we will work with a Lyapunov function of this kind in Theorem 2 below.

Lemma 2 Suppose that there exists a $C^{1}$ function $V_{2}: \mathbb{R}^{k} \rightarrow \mathbb{R}$, class $\mathcal{K}_{\infty}$ functions $\alpha_{1, \mu}, \alpha_{2, \mu}, \rho_{\mu}$, and a continuous positive definite function $\alpha_{3, \mu}:[0, \infty) \rightarrow$ $[0, \infty)$ satisfying

$$
\alpha_{1, \mu}(|\mu|) \leq V_{2}(\mu) \leq \alpha_{2, \mu}(|\mu|)
$$

such that we have

$$
\begin{aligned}
V_{2}(\mu) \geq \rho_{\mu}(|x|) \quad \Rightarrow \quad & V_{2}\left(R_{\mu}(x, \mu, \tau)\right)-V_{2}(\mu) \\
& \leq-\alpha_{3, \mu}\left(V_{2}(\mu)\right)
\end{aligned}
$$

and

$$
V_{2}(\mu) \leq \rho_{\mu}(r) \text { and }|x| \leq r \Rightarrow V_{2}\left(R_{\mu}(x, \mu, \tau)\right) \leq \rho_{\mu}(r) .
$$

Suppose also that for each $t>t_{0}$ such that $V_{2}(\mu(s)) \geq \rho_{\mu}\left(\|x\|_{\left[t_{0}, s\right]}\right)$ for all $s \in\left[t_{0}, t\right)$, the number $N\left(t, t_{0}\right)$ of discrete events in the interval $\left[t_{0}, t\right]$ satisfies

$$
N\left(t, t_{0}\right) \geq \eta\left(t-t_{0}\right)
$$

where $\eta$ is an increasing function. Then the $\mu$-subsystem is ISS with respect to $x$, with gain $\gamma_{\mu}:=\alpha_{1, \mu}^{-1} \circ \rho_{\mu}$.

We can take $\alpha_{3, \mu} \in \mathcal{K}_{\infty}$ with no loss of generality [17]. The conditions (9) and (10) are both satisfied if we have

$$
V_{2}\left(R_{\mu}(x, \mu, \tau)\right)-V_{2}(\mu) \leq-\alpha_{4, \mu}\left(V_{2}(\mu)\right)+\chi_{\mu}(|x|)
$$

for some $\alpha_{4, \mu}, \chi_{\mu} \in \mathcal{K}_{\infty}$. Indeed, letting $\rho_{\mu}(r):=\alpha_{4, \mu}^{-1}\left(2 \chi_{\mu}(r)\right)$, we see that $(9)$ holds with $\alpha_{3, \mu}:=\alpha_{4, \mu} / 2$. Decreasing $\alpha_{4, \mu}$ if necessary, assume with no loss of generality that $i d-\alpha_{4, \mu} \in \mathcal{K}$ (cf. [18]). We then have

$V_{2}(\mu) \leq \alpha_{4, \mu}^{-1}\left(2 \chi_{\mu}(r)\right)$ and $|x| \leq r \Rightarrow V_{2}\left(R_{\mu}(x, \mu, \tau)\right) \leq \chi_{\mu}(|x|)+\left(i d-\alpha_{4, \mu}\right)\left(\alpha_{4, \mu}^{-1}\left(2 \chi_{\mu}(r)\right)\right)<\alpha_{4, \mu}^{-1}\left(2 \chi_{\mu}(|x|)\right)$

and so (10) holds with the same $\rho_{\mu}$. Moreover, (9) implies (12)-and consequently (10) - if the map $R_{\mu}$ is continuous at $(x, \mu)=(0,0)$ and does not depend on $\tau$. Still, it is useful to write two separate conditions (9) and (10) if we want the least conservative expression for the ISS gain. The former condition coupled with (11) is the main ingredient for obtaining ISS, while the latter is automatically enforced if, for example, discrete events can only decrease $V_{2}(\mu)$; this is the case in the example of Section 6. An example of a function $\eta$ that can be used in (11) is $\eta(r)=\frac{r}{\delta_{a}}-N_{0}$, where $\delta_{a}$ and $N_{0}$ are positive numbers (see [16]). In this case, (11) says that discrete events must happen at least every $\delta_{a}$ units of time on the average, modulo a finite number of events that can be "missed". 
Proof of Lemma 2. Let

$$
\bar{t}:=\min \left\{t \geq t_{0}: V_{2}(\mu(t)) \leq \rho_{\mu}\left(\|x\|_{\left[t_{0}, t\right]}\right)\right\} \leq \infty
$$

(this is well defined in view of right-continuity). By virtue of (9), we have

$$
V_{2}(\mu)-V_{2}\left(\mu^{-}\right) \leq-\alpha_{3, \mu}\left(V_{2}\left(\mu^{-}\right)\right)
$$

at each event time in the interval $\left[t_{0}, \bar{t}\right)$. Therefore, there exists a function $\bar{\beta} \in \mathcal{K} \mathcal{L}$ such that

$$
V_{2}(\mu(t)) \leq \bar{\beta}\left(V_{2}\left(\mu\left(t_{0}\right)\right), N\left(t, t_{0}\right)\right) \quad \forall t \in\left[t_{0}, \bar{t}\right)
$$

(cf. [18]). Invoking (11), we have

$$
V_{2}(\mu(t)) \leq \bar{\beta}\left(V_{2}\left(\mu\left(t_{0}\right)\right), \eta\left(t-t_{0}\right)\right) \quad \forall t \in\left[t_{0}, \bar{t}\right)
$$

hence

$$
|\mu(t)| \leq \alpha_{1, \mu}^{-1}\left(\bar{\beta}\left(\alpha_{2, \mu}\left(\left|\mu\left(t_{0}\right)\right|\right), \eta\left(t-t_{0}\right)\right)\right)=: \beta_{\mu}\left(\left|\mu\left(t_{0}\right)\right|, t-t_{0}\right) \quad \forall t \in\left[t_{0}, \bar{t}\right)
$$

Next, (10) applied with $r:=\|x\|_{\left[t_{0}, t\right]}$ at each event time guarantees that

$$
V_{2}(\mu(t)) \leq \rho_{\mu}\left(\|x\|_{\left[t_{0}, t\right]}\right) \quad \forall t \geq \bar{t}
$$

hence

$$
|\mu(t)| \leq \alpha_{1, \mu}^{-1} \circ \rho_{\mu}\left(\|x\|_{\left[t_{0}, t\right]}\right) \quad \forall t \geq \bar{t}
$$

Combining the two bounds for $|\mu(t)|$ gives the desired estimate.

Remark 1 If the second inequality in (9) holds always, i.e., the hypotheses of Lemma 2 are satisfied with $\rho_{\mu}=0$, then the proof shows that the $\mu$-subsystem is $\operatorname{GAS}\left(\gamma_{\mu}=0\right)$.

\section{Lyapunov-based small-gain theorem}

Consider again the hybrid system $\mathcal{H}$ defined in Section 2.1 and decomposed as in Figure 1(a). Here we assume for simplicity that $R_{x}(x, \mu, \tau) \equiv x$ (continuous state does not jump at the event times). Theorem 1, applied to this special feedback decomposition, provides sufficient conditions for ISS. The proof of this theorem is based on trajectory analysis. Lemmas 1 and 2 can be used to check the hypotheses of Theorem 1, and involve ISS-Lyapunov functions for the two subsystems. The question naturally arises whether Theorem 1 can be formulated and proved entirely in terms of such Lyapunov functions. It is known that such an alternative formulation is possible for continuous-time as well as discretetime small-gain theorems $[4,5]$, but this issue has not been pursued for hybrid systems.

Here we present a preliminary result in this direction. We denote by $t_{k}$, $k=1,2, \ldots$ the discrete event times, which we assume to be distinct (with 
no significant changes, we could allow finitely many discrete events to occur simultaneously). It is also convenient to introduce a special clock variable $\bar{\tau}$, which counts the time since the most recent discrete event and is reset to 0 at the event times: $\bar{\tau}(t):=t-t_{k}$ for $t \in\left[t_{k}, t_{k+1}\right)$. It must be noted that the Lyapunov function $V$ constructed in Theorem 2 below depends, besides $x$ and $\mu$, on this variable $\bar{\tau}$. Therefore, it can really be viewed as a Lyapunov function only if the sequence $\left\{t_{k}\right\}$ is independent of the initial state. Otherwise, the proof of ISS using this function is actually a trajectory-based argument (but it still represents an interesting alternative to a purely time-domain one).

Theorem 2 Suppose that there exist positive definite, radially unbounded $C^{1}$ functions $V_{1}: \mathbb{R}^{n} \rightarrow \mathbb{R}$ and $V_{2}: \mathbb{R}^{k} \rightarrow \mathbb{R}$, class $\mathcal{K}_{\infty}$ functions $\chi_{1}, \chi_{2}, \sigma$, and positive constants $b_{1}, b_{2}, c, d, T$ such that we have

$$
\begin{gathered}
V_{1}(x) \geq \chi_{1}\left(V_{2}(\mu)\right) \vee \sigma(|w|) \Rightarrow \nabla V_{1}(x) f(x, \mu, w) \leq-c V_{1}(x), \\
V_{2}(\mu) \geq \chi_{2}\left(V_{1}(x)\right) \Rightarrow V_{2}\left(R_{\mu}(x, \mu, \tau)\right) \leq e^{-d} V_{2}(\mu), \\
V_{2}(\mu) \leq e^{b_{2}} \chi_{2}\left(e^{b_{1}} V_{1}(x)\right) \Rightarrow V_{2}\left(R_{\mu}(x, \mu, \tau)\right) \leq \chi_{2}\left(V_{1}(x)\right)
\end{gathered}
$$

the small-gain condition

$$
e^{b_{1}} \chi_{1}\left(e^{b_{2}} \chi_{2}(r)\right)<r \quad \forall r>0
$$

holds, and the discrete events satisfy

$$
t_{k+1}-t_{k} \leq T \quad \forall k \geq 0 .
$$

Then there exist a locally Lipschitz function $V:[0, T] \times \mathbb{R}^{n} \times \mathbb{R}^{k} \rightarrow \mathbb{R}$, class $\mathcal{K}_{\infty}$ functions $\alpha_{1}, \alpha_{2}, \bar{\sigma}$, a continuous positive definite function $\alpha_{3}:[0, \infty) \rightarrow[0, \infty)$, and a continuous function $\alpha_{4}:[0, T] \times[0, \infty) \rightarrow[0, \infty)$ satisfying $\alpha_{4}(\bar{\tau}, r)>0$ when $\bar{\tau} r \neq 0$, such that for all $\bar{\tau} \in[0, T]$ and all $(x, \mu) \in \mathbb{R}^{n} \times \mathbb{R}^{k}$ the bound

$$
\alpha_{1}(|(x, \mu)|) \leq V(\bar{\tau}, x, \mu) \leq \alpha_{2}(|(x, \mu)|)
$$

holds and we have

$V(\bar{\tau}, x, \mu) \geq \bar{\sigma}(|w|) \Rightarrow \dot{V}(\bar{\tau}, x, \mu):=\frac{\partial V}{\partial \bar{\tau}}(\bar{\tau}, x, \mu)+\frac{\partial V}{\partial x}(\bar{\tau}, x, \mu) f(x, \mu, w) \leq-\alpha_{3}(|(x, \mu)|)$

for the continuous dynamics ${ }^{6}$ and

$$
V\left(0, x, R_{\mu}(x, \mu, \tau)\right)-V(\bar{\tau}, x, \mu) \leq-\alpha_{4}(\bar{\tau},|(x, \mu)|)
$$

for the discrete events. Consequently, $\mathcal{H}$ is ISS with respect to $w$.

\footnotetext{
${ }^{6}$ We will define $V$ as a maximum of two $C^{1}$ functions, hence the gradient $\partial V / \partial x$ is in general not defined at the points where these two functions are equal. At these points, the second term in the definition of $\dot{V}$ is to be understood as the directional derivative of $V$ along $f$. Alternatively, one can use a regularization technique to obtain a smooth Lyapunov function from the locally Lipschitz function $V$; cf. [4].
} 
In spirit, the hypotheses of Theorem 2 match the hypotheses of Theorem 1 and Lemmas 1 and 2, although there are some differences. We note that the condition (17) can be written as $N(t, s) \geq \frac{t-s}{T}$ for all $t>s \geq t_{0}$, i.e., it is a strengthened version of (11). For simplicity, we assumed in (13) and (14) that $V_{1}$ and $V_{2}$ decay at exponential rates. This assumption can be removed, and the more general construction is sketched later in Remark 3 . In the special case when the gain functions $\chi_{1}$ and $\chi_{2}$ are also linear, $b_{1}$ and $b_{2}$ in (15) and (16) can be set to 0 . Note also that (13) only needs to hold for those states where we have continuous evolution, i.e., where $G(x, \mu, \tau)<0$, while (14) and (15) only need to hold for those states where discrete events occur, i.e., where $G(x, \mu, \tau) \geq 0$.

Proof of Theorem 2. We have that $V_{1}$ stays constant during the discrete events while $V_{2}$ stays constant along the continuous dynamics. First, we want to construct modified functions $\bar{V}_{1}$ and $\bar{V}_{2}$ which strictly decrease during the discrete events and the continuous dynamics, respectively, while also enjoying decreasing properties similar to (13)-(15). Pick a number $L_{1} \in\left(0, c \wedge\left(b_{1} / T\right)\right)$ and define

$$
\bar{V}_{1}(\bar{\tau}, x):=e^{L_{1} \bar{\tau}} V_{1}(x) .
$$

Using (17), we have

$$
V_{1}(x) \leq \bar{V}_{1}(\bar{\tau}(t), x) \leq e^{L_{1} T} V_{1}(x) \quad \forall t, x
$$

Similarly, pick a number $L_{2} \in\left(0,\left(d \wedge b_{2}\right) / T\right)$ and define

$$
\bar{V}_{2}(\bar{\tau}, \mu):=e^{-L_{2} \bar{\tau}} V_{2}(\mu)
$$

to obtain

$$
e^{-L_{2} T} V_{2}(\mu) \leq \bar{V}_{2}(\bar{\tau}(t), \mu) \leq V_{2}(\mu) \quad \forall t, \mu .
$$

Define $\bar{\chi}_{1}(r):=e^{L_{1} T} \chi_{1}\left(e^{L_{2} T} r\right)$ and $\bar{\sigma}(r):=e^{L_{1} T} \sigma(r)$. Combining (13), (21), (22), and (24), we have for the continuous dynamics

$\bar{V}_{1}(\bar{\tau}, x) \geq \bar{\chi}_{1}\left(\bar{V}_{2}(\bar{\tau}, \mu)\right) \vee \bar{\sigma}(|w|) \Rightarrow \frac{\partial \bar{V}_{1}}{\partial \bar{\tau}}(\bar{\tau}, x)+\frac{\partial \bar{V}_{1}}{\partial x}(\bar{\tau}, x) f(x, \mu, w) \leq-\left(c-L_{1}\right) \bar{V}_{1}(\bar{\tau}, x)$

and for the discrete events

$$
\bar{V}_{1}(0, x)=e^{-L_{1} \bar{\tau}} \bar{V}_{1}(\bar{\tau}, x) .
$$

Similarly, the evolution of $\bar{V}_{2}$ satisfies

$$
\begin{gathered}
\frac{\partial \bar{V}_{2}}{\partial \bar{\tau}}(\bar{\tau}, \mu)=-L_{2} \bar{V}_{2}(\bar{\tau}, \mu), \\
\bar{V}_{2}(\bar{\tau}, \mu) \geq \chi_{2}\left(\bar{V}_{1}(\bar{\tau}, x)\right) \Rightarrow \bar{V}_{2}\left(0, R_{\mu}(x, \mu, \tau)\right) \leq e^{-\left(d-L_{2} T\right)} \bar{V}_{2}(\bar{\tau}, \mu), \\
\bar{V}_{2}(\bar{\tau}, \mu) \leq \chi_{2}\left(\bar{V}_{1}(\bar{\tau}, x)\right) \Rightarrow \bar{V}_{2}\left(0, R_{\mu}(x, \mu, \tau)\right) \leq \chi_{2}\left(\bar{V}_{1}(\bar{\tau}, x)\right)
\end{gathered}
$$


The condition (16) implies $\bar{\chi}_{1} \circ \chi_{2}(r)<r$ for all $r>0$, which is equivalent to $\chi_{2}(r)<\bar{\chi}_{1}^{-1}(r)$ for all $r>0$. As in [4], pick a $C^{1}$, class $\mathcal{K}_{\infty}$ function $\rho$ with

$$
\rho^{\prime}(r)>0 \quad \forall r>0
$$

such that

$$
\chi_{2}(r)<\rho(r)<\bar{\chi}_{1}^{-1}(r) \quad \forall r>0 .
$$

We are now ready to define a (time-varying) candidate ISS-Lyapunov function for the closed-loop system $\mathcal{H}$ as

$$
V(\bar{\tau}, x, \mu):= \begin{cases}\rho\left(\bar{V}_{1}(\bar{\tau}, x)\right) & \text { if } \rho\left(\bar{V}_{1}(\bar{\tau}, x)\right) \geq \bar{V}_{2}(\bar{\tau}, \mu) \\ \bar{V}_{2}(\bar{\tau}, \mu) & \text { if } \rho\left(\bar{V}_{1}(\bar{\tau}, x)\right)<\bar{V}_{2}(\bar{\tau}, \mu)\end{cases}
$$

We claim that it satisfies (18)-(20). To prove this, pick arbitrary $\bar{\tau} \in[0, T]$ and $(x, \mu) \neq(0,0)$. Let us first consider the case when $V(\bar{\tau}, x, \mu) \geq \bar{\sigma}(|w|)$. We further distinguish between the following two cases.

Case 1: $\rho\left(\bar{V}_{1}(\bar{\tau}, x)\right) \geq \bar{V}_{2}(\bar{\tau}, \mu)$, so that $V(\bar{\tau}, x, \mu)=\rho\left(\bar{V}_{1}(\bar{\tau}, x)\right)$. If $\rho\left(\bar{V}_{1}(\bar{\tau}, x)\right)>$ $\bar{V}_{2}(\bar{\tau}, \mu)$, then we have, using (25), (30), (31), and positive definiteness of $V_{1}$ and $V_{2}$, that $x \neq 0$ and

$\dot{V}(\bar{\tau}, x, \mu)=\rho^{\prime}\left(\bar{V}_{1}(\bar{\tau}, x)\right)\left(\frac{\partial \bar{V}_{1}}{\partial \bar{\tau}}(\bar{\tau}, x)+\frac{\partial \bar{V}_{1}}{\partial x}(\bar{\tau}, x) f(x, \mu, w)\right) \leq-\rho^{\prime}\left(\bar{V}_{1}(\bar{\tau}, x)\right)\left(c-L_{1}\right) \bar{V}_{1}(\bar{\tau}, x)<0$.

If $\rho\left(\bar{V}_{1}(\bar{\tau}, x)\right)=\bar{V}_{2}(\bar{\tau}, \mu)$, then by positive definiteness of $V_{1}$ and $V_{2}$ both $x$ and $\mu$ are nonzero and, invoking also (27), we have

$$
\begin{aligned}
\dot{V}(\bar{\tau}, x, \mu) & =\rho^{\prime}\left(\bar{V}_{1}(\bar{\tau}, x)\right)\left(\frac{\partial \bar{V}_{1}}{\partial \bar{\tau}}(\bar{\tau}, x)+\frac{\partial \bar{V}_{1}}{\partial x}(\bar{\tau}, x) f(x, \mu, w)\right) \vee \frac{\partial \bar{V}_{2}}{\partial \bar{\tau}}(\bar{\tau}, \mu) \\
& \leq-\rho^{\prime}\left(\bar{V}_{1}(\bar{\tau}, x)\right)\left(c-L_{1}\right) \bar{V}_{1}(\bar{\tau}, x) \vee-L_{2} \bar{V}_{2}(\bar{\tau}, \mu)<0
\end{aligned}
$$

Turning to the discrete events, we have three possible cases. If $\rho\left(\bar{V}_{1}(0, x)\right) \geq$ $\bar{V}_{2}\left(0, R_{\mu}(x, \mu, \tau)\right)$, then from (26) we have $V\left(0, x, R_{\mu}(x, \mu, \tau)\right)=\rho\left(\bar{V}_{1}(0, x)\right)=$ $\rho\left(e^{-L_{1} \bar{\tau}} \bar{V}_{1}(\bar{\tau}, x)\right) \leq \rho\left(\bar{V}_{1}(\bar{\tau}, x)\right)=V(\bar{\tau}, x, \mu)$, and the inequality is strict when $\bar{\tau}>0$. If $\rho\left(\bar{V}_{1}(0, x)\right)<\bar{V}_{2}\left(0, R_{\mu}(x, \mu, \tau)\right)$ and $\bar{V}_{2}(\bar{\tau}, \mu) \geq \chi_{2}\left(\bar{V}_{1}(\bar{\tau}, x)\right)$, then using (28) we have $V\left(0, x, R_{\mu}(x, \mu, \tau)\right)=\bar{V}_{2}\left(0, x, R_{\mu}(x, \mu, \tau)\right)<\bar{V}_{2}(\bar{\tau}, \mu) \leq$ $\rho\left(\bar{V}_{1}(\bar{\tau}, x)\right)=V(\bar{\tau}, x, \mu)$. Finally, if $\rho\left(\bar{V}_{1}(0, x)\right)<\bar{V}_{2}\left(0, R_{\mu}(x, \mu, \tau)\right)$ and $\bar{V}_{2}(\bar{\tau}, \mu) \leq$ $\chi_{2}\left(\bar{V}_{1}(\bar{\tau}, x)\right)$, then with the help of $(29)$ we obtain $V\left(0, x, R_{\mu}(x, \mu, \tau)\right)=\bar{V}_{2}\left(0, x, R_{\mu}(x, \mu, \tau)\right) \leq$ $\chi_{2}\left(\bar{V}_{1}(\bar{\tau}, x)\right)<\rho\left(\bar{V}_{1}(\bar{\tau}, x)\right)=V(\bar{\tau}, x, \mu)$.

Case 2: $\rho\left(\bar{V}_{1}(\bar{\tau}, x)\right)<\bar{V}_{2}(\bar{\tau}, \mu)$, so that $V(\bar{\tau}, x, \mu)=\bar{V}_{2}(\bar{\tau}, \mu)$. Using $(27)$ and positive definiteness of $V_{2}$, we have $\mu \neq 0$ and

$$
\dot{V}(\bar{\tau}, x, \mu)=\frac{\partial \bar{V}_{2}}{\partial \bar{\tau}}(\bar{\tau}, \mu)=-L_{2} \bar{V}_{2}(\bar{\tau}, \mu)<0 .
$$

As for the discrete events, (28) and (31) imply that $\bar{V}_{2}\left(0, R_{\mu}(x, \mu, \tau)\right)<\bar{V}_{2}(\bar{\tau}, \mu)$. If $\bar{V}_{2}\left(0, R_{\mu}(x, \mu, \tau)\right)>\rho\left(\bar{V}_{1}(0, x)\right)$, then $V\left(0, x, R_{\mu}(x, \mu, \tau)\right)=\bar{V}_{2}\left(0, R_{\mu}(x, \mu, \tau)\right)<$ 
$\bar{V}_{2}(\bar{\tau}, \mu) \quad=\quad V(\bar{\tau}, x, \mu)$. On the other hand, if $\bar{V}_{2}\left(0, R_{\mu}(x, \mu, \tau)\right) \leq \rho\left(\bar{V}_{1}(0, x)\right)$, then by virtue of $(26)$ we have $V\left(0, x, R_{\mu}(x, \mu, \tau)\right)=$ $\rho\left(\bar{V}_{1}(0, x)\right) \leq \rho\left(\bar{V}_{1}(\bar{\tau}, x)\right)<\bar{V}_{2}(\bar{\tau}, \mu)=V(\bar{\tau}, x, \mu)$.

Since $V_{1}$ and $V_{2}$ are positive definite and radially unbounded, there exist functions $\alpha_{1, x}, \alpha_{2, x}, \alpha_{1, \mu}, \alpha_{2, \mu} \in \mathcal{K}_{\infty}$ such that (6) and (8) hold. Using (22), (24), and (32), we obtain

$$
\rho\left(\alpha_{1, x}(|x|) \vee e^{-L_{2} T} \alpha_{1, \mu}(|\mu|) \leq V(\bar{\tau}, x, \mu) \leq \rho\left(e^{L_{1} T} \alpha_{2, x}(|x|) \vee \alpha_{2, \mu}(|\mu|) .\right.\right.
$$

It is now a routine exercise to construct functions $\alpha_{1}, \alpha_{2} \in \mathcal{K}_{\infty}$ for which (18) holds. Next, observe that the condition $V(\bar{\tau}, x, \mu) \geq \bar{\sigma}(|w|)$ was used, via (25), only to prove the decrease of $V$ along the continuous dynamics but not during the discrete events. Thus (19) and (20) are established (constructing $\alpha_{3}$ and $\alpha_{4}$ is again a simple exercise). Finally, ISS of $\mathcal{H}$ with respect to $w$ follows from (18)(20) via standard arguments (cf. $[3,16])$.

Remark 2 ISS of $\mathcal{H}$ would still hold if we relaxed (20) to just $V\left(0, x, R_{\mu}(x, \mu, \tau)\right) \leq$ $V(\bar{\tau}, x, \mu)$, leaving all the other hypotheses of Theorem 2 unchanged. To construct a function $V$ with these weaker properties, we could set $L_{1}=0$ in the above proof, i.e., work with the original function $V_{1}$ in place of $\bar{V}_{1}$; accordingly, we could set $b_{1}=0$, and also the linearity of the right-hand side of (13) in $V_{1}$ would not be important (see also Remark 3 below). On the other hand, the stronger condition (20) makes the Lyapunov function $V$ more useful for quantifying the effect of the discrete events. In particular, if we impose a dwell-time constraint $t_{k+1}-t_{k} \geq \varepsilon>0$ for all $k \geq 0$, then a uniform decrease condition of the form $V-V^{-} \leq-\bar{\alpha}_{4}\left(V^{-}\right)$, with $\bar{\alpha}_{4}$ continuous positive definite, holds for all discrete events, yielding the stronger property of ISS with respect to a "hybrid time domain" in which the continuous time $t$ and the discrete event index $k$ play essentially equivalent roles (see [12]).

Remark 3 If the right-hand side of (13) in nonlinear in $V_{1}$ :

$$
V_{1}(x) \geq \chi_{1}\left(V_{2}(\mu)\right) \vee \sigma(|w|) \Rightarrow \nabla V_{1}(x) f(x, \mu, w) \leq-\alpha_{1}\left(V_{1}(x)\right)
$$

for some $\alpha_{1} \in \mathcal{K}_{\infty}$, then the construction of $\bar{V}_{1}(\bar{\tau}, x)$ needs to be modified. A standard comparison principle gives a class $\mathcal{K} \mathcal{L}$ function $\beta_{1}$ such that $V_{1}(\bar{\tau})=$ $\beta_{1}\left(V_{1}(0), \bar{\tau}\right)$. With no loss of generality, we take it to be of class $\mathcal{K}_{\infty}$ in $r$ (for each fixed $t$ ) and strictly decreasing in $t$ (for each fixed $r$ ). We can use this function to define $\bar{V}_{1}(\bar{\tau}, x(t)):=\beta_{1}\left(V_{1}(x(t-\bar{\tau})), \delta \bar{\tau}\right)$, where $\delta \in(0,1)$. This can be rewritten as $\bar{V}_{1}(\bar{\tau}, x)=\beta_{1}\left(\beta_{1, \bar{\tau}}^{-1}\left(V_{1}(x)\right), \delta \bar{\tau}\right)$, where $\beta_{1, t}(r):=\beta_{1}(r, t)$. It follows from this and (17) that $V_{1}(x) \leq \bar{V}_{1}(\bar{\tau}(t), x) \leq\left(i d+\kappa_{1}\right)\left(V_{1}(x)\right)$ for some positive definite function $\kappa_{1}$. Similarly, if the right-hand side of (14) is given by $\left(i d-\alpha_{2}\right)\left(V_{2}(\mu)\right)$ and we define a function $\bar{V}_{2}(\bar{\tau}, \mu)$ in the same way as $\bar{V}_{1}$ above but using $\beta_{2}$ associated with $\frac{1}{\tau} \alpha_{2}$, then it satisfies $\left(i d-\kappa_{2}\right)\left(V_{2}(\mu)\right) \leq \bar{V}_{2}(\bar{\tau}(t), \mu) \leq V_{2}(\mu)$. The statement of the result and the rest of the proof are then analogous to those of Theorem 2, except that the small-gain condition becomes more complicated 
because it depends on $\kappa_{1}$ and $\kappa_{2}$. (Note that the linear dependence of the righthand sides of (25), (27), and (28) on $\bar{V}_{1}$ and $\bar{V}_{2}$ played no role in the subsequent arguments.)

As an alternative to constructing a Lyapunov function strictly decreasing along solutions, we can work with a weak Lyapunov function non-strictly decreasing along solutions and apply a LaSalle invariance principle for hybrid systems, such as the one proved in [6] (see also [19] for recent generalizations and improvements). As can be seen from the proof of the result given next, such an approach is perhaps simpler and more natural in the situation at hand, and the relevant hypotheses more closely match those of Theorem 1 and Lemmas 1 and 2 . However, the result has inherent limitations characteristic of LaSalle theorems; in particular, it is restricted to disturbance-free, time-invariant dynamics.

Consider the same hybrid system $\mathcal{H}$ as in Theorem 2, but assume that there are no disturbances, i.e., the continuous dynamics are described by $\dot{x}=f(x, \mu)$. We omit also the clock variables $\tau$, so that the discrete events are defined as $G\left(x^{-}, \mu^{-}\right) \geq 0 \Rightarrow \mu=R_{\mu}\left(x^{-}, \mu^{-}\right)$. We assume as before that the resulting discrete event times are distinct (the extension to the case when a finite number of discrete events can occur simultaneously is straightforward). We also assume that the behavior of $\mathcal{H}$ is continuous with respect to initial conditions, in the sense defined and characterized in [6].

Theorem 3 Suppose that there exist positive definite, radially unbounded $C^{1}$ functions $V_{1}: \mathbb{R}^{n} \rightarrow \mathbb{R}$ and $V_{2}: \mathbb{R}^{k} \rightarrow \mathbb{R}$, class $\mathcal{K}_{\infty}$ functions $\chi_{1}, \chi_{2}$, and continuous positive definite functions $\alpha_{1}, \alpha_{2}:[0, \infty) \rightarrow[0, \infty)$ such that we have

$$
\begin{gathered}
V_{1}(x) \geq \chi_{1}\left(V_{2}(\mu)\right) \Rightarrow \nabla V_{1}(x) f(x, \mu) \leq-\alpha_{1}\left(V_{1}(x)\right), \\
V_{2}(\mu) \geq \chi_{2}\left(V_{1}(x)\right) \Rightarrow V_{2}\left(R_{\mu}(x, \mu, \tau)\right)-V_{2}(\mu) \leq-\alpha_{2}\left(V_{2}(\mu)\right), \\
V_{2}(\mu) \leq \chi_{2}\left(V_{1}(x)\right) \Rightarrow V_{2}\left(R_{\mu}(x, \mu, \tau)\right) \leq \chi_{2}\left(V_{1}(x)\right)
\end{gathered}
$$

the small-gain condition

$$
\chi_{1} \circ \chi_{2}(r)<r \quad \forall r>0
$$

holds, and for each $t>t_{0}$ such that $V_{2}(\mu(s)) \geq \chi_{2}\left(V_{1}(x(s))\right)$ for all $s \in\left[t_{0}, t\right)$, the number $N\left(t, t_{0}\right)$ of discrete events in the interval $\left[t_{0}, t\right]$ satisfies (11) for some increasing function $\eta$. Then there exists a positive definite, radially unbounded, locally Lipschitz function $V: \mathbb{R}^{n} \times \mathbb{R}^{k} \rightarrow \mathbb{R}$ such that for all $(x, \mu) \in \mathbb{R}^{n} \times \mathbb{R}^{k}$ we have

$$
\dot{V}(x, \mu):=\frac{\partial V}{\partial x}(x, \mu) f(x, \mu) \leq 0
$$

for the continuous dynamics ${ }^{7}$ and

$$
V\left(x, R_{\mu}(x, \mu, \tau)\right) \leq V(x, \mu)
$$

\footnotetext{
${ }^{7}$ When the gradient $\partial V / \partial x$ is not defined, $\dot{V}$ is to be interpreted as the directional derivative of $V$ along $f$; cf. footnote 6 .
} 
for the discrete events. Moreover, there is no (forward) invariant set other than the origin inside the set $S_{1} \cup S_{2}$, where

$$
S_{1}:=\{(x, \mu): \dot{V}(x, \mu)=0, G(x, \mu)<0\}
$$

and

$$
S_{2}:=\left\{(x, \mu): V\left(x, R_{\mu}(x, \mu, \tau)\right)=V(x, \mu), G(x, \mu) \geq 0\right\} .
$$

Consequently, $\mathcal{H}$ is GAS.

As in Theorem 2, the condition (33) only needs to be true for those states where we have continuous evolution, i.e., where $G(x, \mu)<0$, while (34) and (35) only need to be true for those states where discrete events occur, i.e., where $G(x, \mu) \geq 0$.

Proof of Theorem 3. The condition (36) is equivalent to $\chi_{2}(r)<\chi_{1}^{-1}(r)$ for all $r>0$. As in [6], pick a $C^{1}$, class $\mathcal{K}_{\infty}$ function $\rho$ satisfying (30) and

$$
\chi_{2}(r)<\rho(r)<\chi_{1}^{-1}(r) \quad \forall r>0 .
$$

Define a candidate weak Lyapunov function for $\mathcal{H}$ as

$$
V(x, \mu):= \begin{cases}\rho\left(V_{1}(x)\right) & \text { if } \rho\left(V_{1}(x)\right) \geq V_{2}(\mu) \\ V_{2}(\mu) & \text { if } \rho\left(V_{1}(x)\right)<V_{2}(\mu)\end{cases}
$$

This function is positive definite and radially unbounded by construction. We now prove that it satisfies (37) and (38). We consider two cases, similarly to the proof of Theorem 2.

Case 1: $\rho\left(V_{1}(x)\right) \geq V_{2}(\mu)$, so that $V(x, \mu)=\rho\left(V_{1}(x)\right)$. If $\rho\left(V_{1}(x)\right)>V_{2}(\mu)$, then we have, using (30), (33), (39), and positive definiteness of $V_{1}$ and $V_{2}$, that $x \neq 0$ and

$$
\dot{V}(x, \mu)=\rho^{\prime}\left(V_{1}(x)\right) \frac{\partial V_{1}}{\partial x}(x) f(x, \mu) \leq-\rho^{\prime}\left(V_{1}(x)\right) \alpha_{1}\left(V_{1}(x)\right)<0 .
$$

If $\rho\left(V_{1}(x)\right)=V_{2}(\mu)$ then, since $V_{2}$ stays constant along the continuous dynamics, we have

$$
\dot{V}(x, \mu) \leq-\rho^{\prime}\left(V_{1}(x)\right) \alpha_{1}\left(V_{1}(x)\right) \vee 0 \leq 0 .
$$

We know that the discrete events do not change the value of $\rho\left(V_{1}(x)\right)$. If $V_{2}(\mu) \geq$ $\chi_{2}\left(V_{1}(x)\right)$, then using (34) we have $V_{2}\left(x, R_{\mu}(x, \mu, \tau)\right) \leq V_{2}(\mu) \leq \rho\left(V_{1}(x)\right)$. If $V_{2}(\mu) \leq \chi_{2}\left(V_{1}(x)\right)$, then with the help of (35) we obtain $V_{2}\left(x, R_{\mu}(x, \mu, \tau)\right) \leq$ $\chi_{2}\left(V_{1}(x)\right) \leq \rho\left(V_{1}(x)\right)$. In either case we have $V_{2}\left(R_{\mu}(x, \mu, \tau)\right) \leq \rho\left(V_{1}(x)\right)$, hence $V\left(x, R_{\mu}(x, \mu, \tau)\right)=\rho\left(V_{1}(x)\right)=V(x, \mu)$.

Case 2: $\rho\left(V_{1}(x)\right)<V_{2}(\mu)$, so that $V(x, \mu)=V_{2}(\mu)$. For the continuous dynamics, we have $\dot{V}(x, \mu)=0$. As for the discrete events, (34) and (39) imply that $V_{2}\left(R_{\mu}(x, \mu, \tau)\right)<V_{2}(\mu)$. If $V_{2}\left(R_{\mu}(x, \mu, \tau)\right)>\rho\left(V_{1}(x)\right)$, then $V\left(x, R_{\mu}(x, \mu, \tau)\right)=$ $V_{2}\left(R_{\mu}(x, \mu, \tau)\right)<V_{2}(\mu)=V(x, \mu)$. If $V_{2}\left(R_{\mu}(x, \mu, \tau)\right) \leq \rho\left(V_{1}(x)\right)$, then we have $V\left(x, R_{\mu}(x, \mu, \tau)\right)=\rho\left(V_{1}(x)\right)<V_{2}(\mu)=V(x, \mu)$. 
The properties (37) and (38) are therefore established. Next, we turn to the claim about the absence of a nonzero invariant set inside $S_{1} \cup S_{2}$. The previous analysis implies that we have $S_{1} \subseteq \tilde{S}_{1}$ and $S_{2} \subseteq \tilde{S}_{2}$, where

$$
\tilde{S}_{1}:=\left\{(x, \mu): \rho\left(V_{1}(x)\right) \leq V_{2}(\mu), G(x, \mu)<0\right\}
$$

and

$$
\tilde{S}_{2}:=\left\{(x, \mu): \rho\left(V_{1}(x)\right) \geq V_{2}(\mu), G(x, \mu) \geq 0\right\} .
$$

Hence it is enough to prove the claim for $\tilde{S}_{1} \cup \tilde{S}_{2}$. By (39) and the hypotheses placed on the discrete events, no subset of either $\tilde{S}_{1}$ or $\tilde{S}_{2}$ can be invariant. Indeed, while the state is in $\tilde{S}_{1},(11)$ holds and so a discrete event must eventually occur, which means that the state must leave $\tilde{S}_{1}$. On the other hand, since consecutive discrete events are assumed to be separated by positive intervals of continuous evolution, $\tilde{S}_{2}$ is not invariant. It remains to show that discrete events cannot take the state from $\tilde{S}_{2} \backslash\{(0,0)\}$ to $\tilde{S}_{1}$. Consider an arbitrary $(x, \mu) \in \tilde{S}_{2} \backslash\{(0,0)\}$. If $V_{2}(\mu) \geq \chi_{2}\left(V_{1}(x)\right)$, then from (34) we have $V_{2}\left(x, R_{\mu}(x, \mu, \tau)\right)<V_{2}(\mu) \leq \rho\left(V_{1}(x)\right)$. If $V_{2}(\mu) \leq \chi_{2}\left(V_{1}(x)\right)$, then from (35) we have $V_{2}\left(x, R_{\mu}(x, \mu, \tau)\right) \leq \chi_{2}\left(V_{1}(x)\right)<\rho\left(V_{1}(x)\right)$. We conclude that $\left(x, R_{\mu}(x, \mu)\right)$ cannot be in $\tilde{S}_{1}$, which establishes the claim.

Stability in the sense of Lyapunov and boundedness of all solutions follow from (37), (38), and the fact that $V$ is positive definite and radially unbounded. Since $\mathcal{H}$ is non-blocking and deterministic by construction, the invariance principle for hybrid systems from [6] applies. To conclude GAS, we need to rule out the existence of an invariant set different from the origin inside the set on which $V$ does not strictly decrease. But this latter set is precisely $S_{1} \cup S_{2}$, hence the proof is complete.

We see that although the function $V$ in Theorem 3 is a weak Lyapunov function, it has the right properties for applying the LaSalle invariance principle and concluding GAS. However, for other purposes (such as, for example, analyzing stability under perturbations of the right-hand side) it is still desirable to have a strictly decreasing Lyapunov function. One may try to construct such a Lyapunov function by modifying $V$ under appropriate "observability" conditions (see, e.g., $[20,21]$ for results of this kind for continuous systems).

\section{Application example: quantized feedback stabilization}

Consider the linear time-invariant system

$$
\dot{x}=A x+B u, \quad x \in \mathbb{R}^{n}, u \in \mathbb{R}^{m}
$$

where $A$ is a non-Hurwitz matrix. We assume that this system is stabilizable, so that there exist matrices $P=P^{T}>0$ and $K$ such that

$$
(A+B K)^{T} P+P(A+B K) \leq-2 I .
$$

We denote by $\lambda_{\min }(\cdot)$ and $\lambda_{\max }(\cdot)$ the smallest and the largest eigenvalue of a symmetric matrix, respectively. A quantizer is a piecewise constant function $q$ : 
$\mathbb{R}^{n} \rightarrow \mathcal{Q}$, where $\mathcal{Q}$ is a finite subset of $\mathbb{R}^{n}$, for which there exist positive numbers $M$ (the range of $q$, possibly $\infty$ ) and $\Delta$ (the quantization error) satisfying

$$
|z| \leq M \Rightarrow|q(z)-z| \leq \Delta
$$

It is well known that quantization errors in general destroy asymptotic stability, in the sense that the quantized feedback law $u=K q(x)$ is no longer stabilizing. To overcome this problem, we will use quantized measurements of the form

$$
q_{\mu}(x):=\mu q\left(\frac{x}{\mu}\right), \quad \mu>0 .
$$

The quantizer $q_{\mu}$ has range $M \mu$ and quantization error $\Delta \mu$. The "zoom" variable $\mu$ will be the discrete variable of the hybrid closed-loop system, initialized at some fixed value. The idea behind achieving asymptotic stability is to "zoom in", i.e., decrease $\mu$ to 0 in a suitable discrete fashion, while applying the feedback law $u=K q_{\mu}(x)$. To simplify the exposition, we will assume that the condition $|x| \leq M \mu$ always holds, i.e., $x$ always remains within the range of $q_{\mu}$. This is automatically true if $M$ is infinite. For finite $M$, this can be achieved by incorporating an initial "zooming-out" scheme and subsequently ensuring that the condition is never violated (see [9] for details).

We view the closed-loop system as the feedback interconnection of Figure $1(\mathrm{a})$, with $w \equiv 0$ for the time being. Its continuous dynamics are

$$
\dot{x}=A x+B K q_{\mu}(x)=(A+B K) x+B K \mu\left(q\left(\frac{x}{\mu}\right)-\frac{x}{\mu}\right) .
$$

In view of (42) and the fact that $A+B K$ is Hurwitz, we have ISS with respect to $\mu$. Let us use the Lyapunov function $V(x):=\frac{1}{2} x^{T} P x$, with $P$ from (41), to compute the ISS gain. Its derivative along solutions satisfies

$$
\dot{V} \leq-|x|^{2}+|x|\|P B K\| \Delta \mu
$$

where $\|\cdot\|$ stands for matrix induced norm. A simple square completion argument shows that for an arbitrary $\varepsilon>0$, we have

$$
|x|^{2} \geq(1+\varepsilon)^{2}\|P B K\|^{2} \Delta^{2} \mu^{2} \quad \Rightarrow \quad \dot{V} \leq-\frac{\varepsilon}{1+\varepsilon}|x|^{2} .
$$

It follows that the condition (7) of Lemma 1 holds with $\rho_{x}(r):=\lambda_{\max }(P)(1+$ $\varepsilon)^{2}\|P B K\|^{2} \Delta^{2} r^{2}$, and consequently the $x$-subsystem is ISS with respect to $\mu$, with gain

$$
\gamma_{x}(r):=\sqrt{\frac{\lambda_{\max }(P)}{\lambda_{\min }(P)}}(1+\varepsilon)\|P B K\| \Delta r .
$$

We now need to describe a scheme for updating $\mu$, which we refer to as a quantization protocol. The goal is to guarantee ISS of the $\mu$-subsystem with respect to $x$, with the ISS gain $\gamma_{\mu}$ such that the small-gain condition of Theorem 1 holds. Any such ISS quantization protocol will do; we now give an example. 
Pick a number $C$ satisfying

$$
\sqrt{\lambda_{\min }(P)} C>\sqrt{\lambda_{\max }(P)}\|P B K\| \Delta .
$$

Define the guard map by

$$
G(x, \mu, \tau):=\left(\begin{array}{c}
(C+\Delta) \mu-\left|q_{\mu}(x)\right| \\
\tau-\delta
\end{array}\right)
$$

for some $\delta>0$, where the auxiliary clock variable $\tau$ is scalar-valued. Define the reset map by

$$
R(x, \mu, \tau):=\left(\begin{array}{c}
x \\
\Omega_{i} \mu \\
0
\end{array}\right)
$$

for some $\Omega_{i} \in(0,1)$. In other words, whenever we have $\left|q_{\mu^{-}}\left(x^{-}\right)\right| \leq(C+$ $\Delta) \mu^{-}$and $\tau^{-} \geq \delta$, we set $\mu=\Omega_{i} \mu^{-}$("zoom in") and $\tau=0$ (reset the clock). The system is clearly well posed. In view of (42), it is easy to see that the condition (9) of Lemma 2 is satisfied with $W(\mu):=\mu^{2}$ and $\rho_{\mu}(r):=r^{2} / C^{2}$. The other hypotheses of that lemma are also satisfied by construction. Therefore, the $\mu$-subsystem is ISS with respect to $x$, with gain

$$
\gamma_{\mu}(r):=r / C
$$

We see that both gain functions are actually linear gains, and to apply Theorem 1 we need the product of these gains to be smaller than 1 . Since $\varepsilon$ in (44) can be arbitrarily small, the small-gain condition is exactly (45).

This quantization protocol has a clear geometric interpretation. We zoom in if the quantized measurements show that $|x| \leq(C+2 \Delta) \mu$, which is guaranteed to happen whenever $|x| \leq C \mu$. The condition (45) means that for each $\mu$, the ball of radius $C \mu$ around the origin contains the level set of $V$ superscribed around the ball of radius $\|P B K\| \Delta \mu$, outside of which $V$ is known to decay (thus ensuring that the zoom-in will be triggered). Similar ideas were used in $[8$, 9], but previous analyses did not employ the small-gain argument and were arguably less transparent.

We remark in passing that the choice of precise values for $C$ and $\Omega_{i}$ is also dictated by the need to keep $x$ within the range of $q_{\mu}$. Namely, for each $\mu$, the ball of radius $(C+2 \Delta) \mu$ must be contained in the level set of $V$ inscribed in the ball of radius $M \Omega_{i} \mu$, so we must have

$$
\sqrt{\lambda_{\max }(P)}(C+2 \Delta) \leq \sqrt{\lambda_{\min }(P)} M \Omega_{i} .
$$

Since these considerations seem to be decoupled from the small-gain argument, we refer the reader to [9] for details.

Several variations of the above scheme are possible. One is a "sampled-data" version, where the zoom-in condition is checked and transition is executed only at integer multiples of $\delta$, i.e., $\tau^{-} \geq \delta$ would be complemented by $-\tau^{-} \geq-\delta$ (cf. the discrete-time design of [8]). We could also just zoom in at each sampling 
time, independently of the quantized measurement, so that the first component of $G$ would be eliminated. Then the $\mu$ subsystem becomes GAS (cf. Remark 1) and the feedback connection reduces to the cascade $\left(\gamma_{\mu}=0\right)$; on the other hand, this scheme involves more frequent discrete transitions and requires more care in choosing $\Omega_{i}$. This variant is pursued in [22].

Nonlinear quantized control systems can also be treated in an entirely similar way, under the assumption of ISS of the continuous closed-loop dynamics with respect to measurement errors. In particular, the quantization protocol for nonlinear systems proposed in [9] lends itself to a small-gain interpretation along the above lines, except that the ISS gains are nonlinear and so the general small-condition (5) must be used.

Probably the most important advantage of the small-gain viewpoint for the quantized feedback problem is that it allows us to establish robustness (in the ISS sense) with respect to external disturbances. This aspect has not been addressed in earlier work and seems to be much more difficult to handle with previously used tools. In the case of infinite quantizer range $M$, the small-gain approach makes the extension immediate. Namely, if we augment the system with an external disturbance $w$ to have

$$
\dot{x}=A x+B u+D w
$$

then Theorem 1 yields ISS of the closed-loop system described above with respect to $w$. If the quantizer range $M$ is finite, then the situation is much more complicated because it is necessary to "zoom out" to keep $x$ within the range of $q_{\mu}$, and in the presence of the disturbance we will keep switching back and forth between the zooming-in and zooming-out stages. A solution to this problem is described in the recent paper [22].

\section{Conclusions and future work}

The main purpose of this paper was to bring the small-gain analysis method to the attention of the hybrid systems community. We argued that general hybrid systems can be viewed as feedback interconnections of simpler subsystems, and thus the small-gain analysis framework is very naturally applicable to them. While the small gain theorem based on time-domain analysis provides an "offthe-shelf" tool for studying stability of hybrid systems, Lyapunov function constructions are also of interest and were addressed in this paper. For a class of hybrid systems satisfying the conditions of the small-gain theorem, we described a construction of a Lyapunov function and another construction of a weak Lyapunov function, each of which can be used to establish stability. An application of the proposed approach to a quantized feedback stabilization problem was shown to lead to useful new interpretations and generalizations of existing results.

Further research is needed for improving Lyapunov function constructions of Section 5, which are currently not quite satisfactory. First, Theorem 2 falls short of recovering the ISS result of Theorem 1. Second, both Theorem 2 and 
Theorem 3 are restricted to the special feedback interconnection shown in Figure 1(a). Another direction for future work is to systematically exploit the proposed method in application-motivated contexts. As demonstrated in [7] (see also [14] and the subsequent work [23]), networked control systems represent a very promising application area, but we expect the small-gain analysis to be useful for hybrid systems arising in many other areas as well.

\section{References}

1. Zames, G.: On the input-output stability of time-varying nonlinear feedback systems, part 1: conditions derived using concepts of loop gain, conicity, and positivity. IEEE Trans. Automat. Control 11 (1966) 228-238

2. Jiang, Z.P., Teel, A.R., Praly, L.: Small-gain theorem for ISS systems and applications. Math. Control Signals Systems 7 (1994) 95-120

3. Sontag, E.D.: Smooth stabilization implies coprime factorization. IEEE Trans. Automat. Control 34 (1989) 435-443

4. Jiang, Z.P., Mareels, I.M.Y., Wang, Y.: A Lyapunov formulation of the nonlinear small-gain theorem for interconnected ISS systems. Automatica 32 (1996) 12111215

5. Laila, D.S., Nešić, D.: Lyapunov based small-gain theorem for parameterized discrete-time interconnected ISS systems. In: Proc. 41st IEEE Conf. on Decision and Control. (2002) 2292-2297

6. Lygeros, J., Johansson, K.H., Simić, S.N., Zhang, J., Sastry, S.S.: Dynamical properties of hybrid automata. IEEE Trans. Automat. Control 48 (2003) 2-17

7. Nešić, D., Liberzon, D.: A small-gain approach to stability analysis of hybrid systems. In: Proc. 44th IEEE Conf. on Decision and Control. (2005) To appear.

8. Brockett, R.W., Liberzon, D.: Quantized feedback stabilization of linear systems. IEEE Trans. Automat. Control 45 (2000) 1279-1289

9. Liberzon, D.: Hybrid feedback stabilization of systems with quantized signals. Automatica 39 (2003) 1543-1554

10. van der Schaft, A., Schumacher, H.: An Introduction to Hybrid Dynamical Systems. Springer, London (2000)

11. Lynch, N., Segala, R., Vaandrager, F.: Hybrid I/O automata. Information and Computation 185 (2003) 105-157

12. Goebel, R., Hespanha, J.P., Teel, A.R., Cai, C., Sanfelice, R.: Hybrid systems: generalized solutions and robust stability. In: Proc. 6th IFAC Symp. on Nonlinear Control Systems (NOLCOS). (2004) 1-12

13. Khalil, H.K.: Nonlinear Systems. 3rd edn. Prentice Hall, New Jersey (2002)

14. Nešić, D., Teel, A.R.: Input-output stability properties of networked control systems. IEEE Trans. Automat. Control 49 (2004) 1650-1667

15. Sontag, E.D., Wang, Y.: Notions of input to output stability. Systems Control Lett. 38 (1999) 235-248

16. Hespanha, J.P., Liberzon, D., Teel, A.R.: On input-to-state stability of impulsive systems. In: Proc. 44th IEEE Conf. on Decision and Control. (2005) To appear.

17. Jiang, Z.P., Wang, Y.: A converse Lyapunov theorem for discrete-time systems with disturbances. Systems Control Lett. 45 (2002) 49-58

18. Jiang, Z.P., Wang, Y.: Input-to-state stability for discrete-time nonlinear systems. Automatica 37 (2001) 857-869 
19. Sanfelice, R.G., Goebel, R., Teel, A.R.: Invariance principles for hybrid systems with connections to detectability and asymptotic stability. (2005) Submitted. Preliminary version appeared as "Results on convergence in hybrid systems via detectability and an invariance principle" in Proc. 2005 American Control Conf.

20. Angeli, D.: Input-to-state stability of PD-controlled robotic systems. Automatica 35 (1999) 1285-1290

21. Mazenc, F., Nešić, D.: Strong Lyapunov functions for systems satisfying the conditions of La Salle. IEEE Trans. Automat. Control 49 (2004) 1026-1030

22. Liberzon, D., Nešić, D.: Input-to-state stabilization of linear systems with quantized feedback. In: Proc. 44th IEEE Conf. on Decision and Control. (2005) To appear.

23. Carnevale, D., Teel, A.R., Nešić, D.: A Lyapunov proof of an improved maximum allowable transfer interval for networked control systems. (IEEE Trans. Automat. Control) Submitted. 